\title{
Study of Langmuir Monolayers of Arachidic Acid on Saline Solutions Using Sum-frequency Generation Vibrational Spectroscopy
}

\author{
Nguyen Thi Hue ${ }^{1,2}$, Vu Thi Thanh Tam ${ }^{1}$, Nguyen Anh Tuan ${ }^{1, *}$ \\ ${ }^{1}$ Faculty of Physics, VNU University of Science, 334 Nguyen Trai, Hanoi, Vietnam \\ ${ }^{2}$ Hung Vuong University, Phu Tho, Vietnam \\ Received 13 March 2017 \\ Revised 28 April 2017; Accepted 25 May 2017
}

\begin{abstract}
In this report, we studied the effect of halide salt concentration on Arachidic Acid (AA) Langmuir monolayers formed on salt solutions, such as $\mathrm{NaCl}, \mathrm{NaI}, \mathrm{NaF}$ by using a SumFrequency Generation Vibrational Spectroscopy. In the SFG spectra, we observed the peak intensities of $\mathrm{CH}_{3 \mathrm{FR}}$ and $\mathrm{CH}_{3 \mathrm{SS}}$ vibrational modes decreased when increasing the salt concentration. This observation indicates that the structure of the AA monolayer was disordered by the interaction of the dissolved ions with the carboxyl head groups of the monolayer.
\end{abstract}

Keywords: Langmuir monolayer, interfacial structure, Sum-frequency vibrational spectroscopy.

\section{Introduction}

Langmuir monolayer is a single monomolecular layer formed on the air/liquid interface. Structures and properties of Langmuir monolayers are often affected by external conditions such as temperature, dissolved ion concentration, and $\mathrm{pH}$. The fatty acid molecules have both hydrophilic and hydrophobic parts. The head group which contains hydrophilic compounds is attracted to water. The tail group of the hydrocarbon chain is a hydrophobic part, which is out of the water and forms a single layer of molecules on the water surface [1]. Langmuir monolayers have attracted studies because their structural resembles biological membranes. The interaction in the interface comes from hydrogenbonding between the head groups and the water molecules. In addition, the presence of dissolved ions affects molecular orientation and structure of the interfacial layers.

Sum-frequency generation vibrational spectroscopy (SFG-VS) is a second-order nonlinear optical process. Within the dipole approximation, SFG is forbidden in centrosymmetric media. Thus, SFG has an intrinsic surface/interfacial selectivity. Recently, thanks to the development of ultrafast laser sources, SFG-VS has become a powerful tool to study dynamic phenomena at surfaces and interfaces [2].

\footnotetext{
*Corresponding author. Tel.: 84-919148855.

Email: tuanphysics@vnu.edu.vn

https://doi.org/10.25073/2588-1124/vnumap.4202
} 
In this study, we used the SFG spectroscopy to investigate the effect of halide anions on the molecular structure of an Arachidic Acid (AA) monolayer on the air-water interface. We have taken SF spectra from AA monolayers on saline solutions of $\mathrm{NaCl}, \mathrm{NaI}$, and $\mathrm{NaF}$ with various concentrations. The effects of those anions have been observed in specific vibrational ranges.

\section{Experiment}

\subsection{Sample preparing}

Arachidic acid solutions $(1 \mathrm{mg} / \mathrm{ml})$ were prepared by dissolving solid-state AA $\left(\mathrm{CH}_{3}\left(\mathrm{CH}_{2}\right)_{18} \mathrm{COOH}\right)$ (Sigma - Aldrich) in chloroform (Sigma - Aldrich). The saline solutions were prepared from sodium chloride (> 99\% purity, Merck), sodium iodide (> 99\% purity, Merck) and sodium fluoride (> 99\% purity, Merck) dissolved in deionized water $(\mathrm{pH} \sim 5.7)$. The AA Langmuir monolayer was formed in 10 minutes after spreading the AA solution on the surface of the saline solutions. We controlled surface areas of the samples in order to maintain a molecular area of $20.4 \AA^{2}$ per molecule, which corresponds to the liquid-condensed phase (LC) of the monolayers [3].

\subsection{Optical setup}

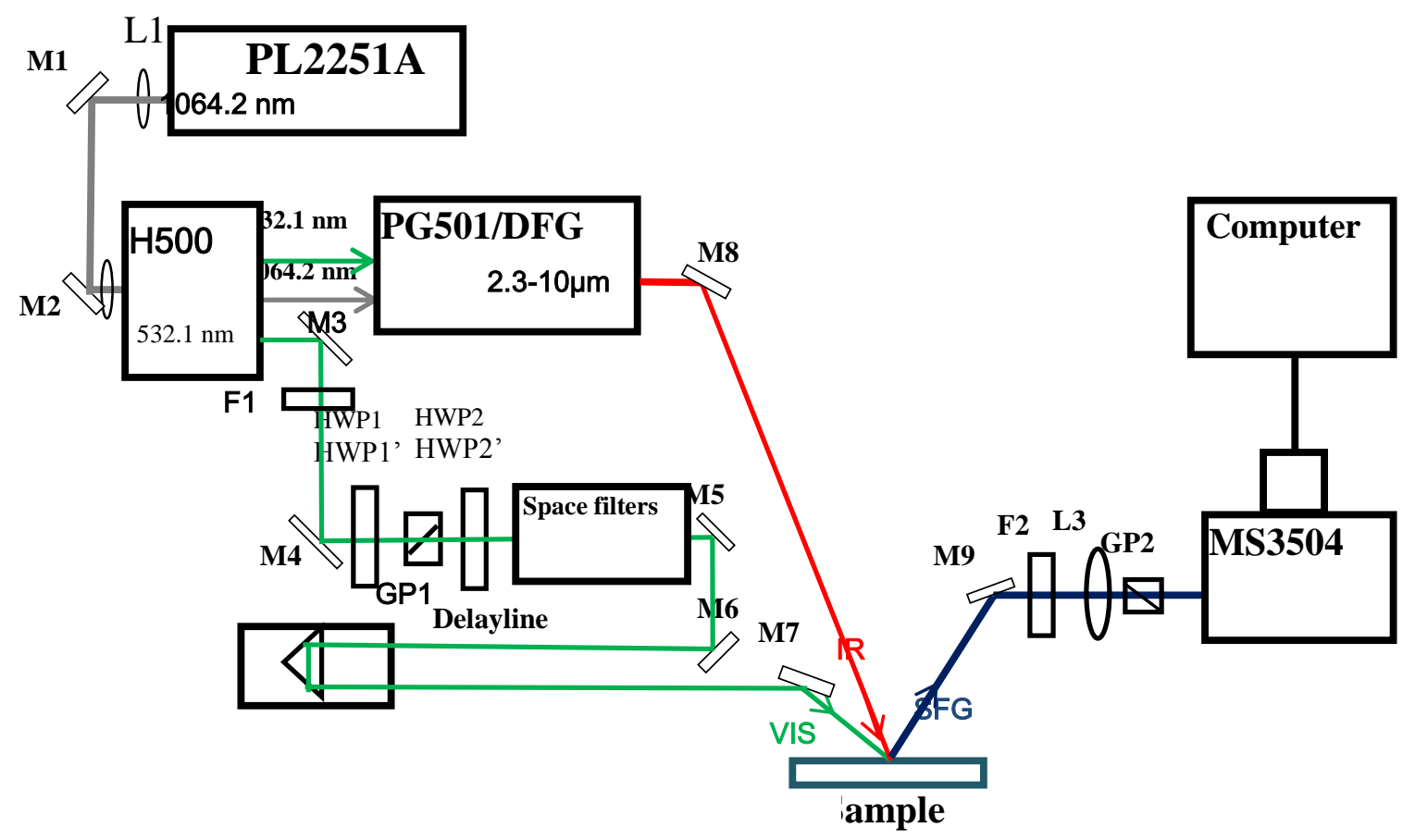
M1-M9: Mirrors GP1;
GP2: Glan prism;
GP2: Glan p
P1: Prism;
L1, L2,L3: Lenses
HWP1, HWP2 - Half wave plates
F1, F2: Filters;

Figure 1. SFG spectroscopy measurement setup. 
The optical setup of our SFG spectrometer is shown in figure 1. In this setup, we used a modelocked Nd:YAG pico-second laser (EKSPLA - PL2251A) as a pump source with wavelength of $1064.2 \mathrm{~nm}$, energy of $50 \mathrm{~mJ} /$ pulse, pulse width of $30 \mathrm{ps}$, and repetition rate of $50 \mathrm{~Hz}$. The fundamental beam of $1064.2 \mathrm{~nm}$ was directed into a second-harmonic unit (H500). The second-harmonic at 532.1 $\mathrm{nm}$ and the fundamental beam at $1064.2 \mathrm{~nm}$ were used to pump an OPG/OPA/DFG system (EKSPLA - PG501). In the DFG block, the $1064.2 \mathrm{~nm}$ laser pulses are mixed with idler waves from the OPG/OPA block in a $\mathrm{AgGaS}_{2}$ crystal to obtain tunable waves covering a mid-infrared range from 2.3$10 \mu \mathrm{m}$. Mirrors M7 and M8 guide the visible $(532.1 \mathrm{~nm})$ and the IR beams into the sample at incident angles of $60^{\circ}$ and $55^{\circ}$, respectively. Once the two incident waves satisfied the phase-matching condition, SF signal was generated at the reflection angle of $59.7^{\circ} \pm 0.35^{\circ}$. The SF light was selected by a monochromator (MS3504) and then detected by photomultiplier tubes. We used a delay line in the visible path to ensure a temporal overlap at the sample. All of the spectra were taken in SSP polarization combination.

\section{Results and discussion}

We have taken SFG spectra from AA monolayer/saline solutions of $\mathrm{NaI}, \mathrm{NaCl}$, and $\mathrm{NaF}$ with various saline concentrations. Those spectra, shown in Fig. 2, Fig. 3, and Fig. 4, are dominated by the symmetric stretching mode of $\mathrm{CH}_{3 \mathrm{ss}}$ at $2880 \mathrm{~cm}^{-1}$ and the Fermi resonance of this mode at $2945 \mathrm{~cm}^{-1}$. Besides, the symmetric stretching mode of $\mathrm{CH}_{2 \mathrm{ss}}$ at $2850 \mathrm{~cm}^{-1}$ is minor or hardly observed. The very low intensity of $\mathrm{CH}_{2 \mathrm{ss}}$ in compare to $\mathrm{CH}_{3}$ modes indicates that the AA monolayers have been well formed at LC phase on the surface of solutions [3].

For convenience of discussion, we separate the spectra into two bands depicted in two panel of each figure, in which the $\mathrm{CH}$ vibrational band on the left and the $\mathrm{OH}$ stretching band on the right. The $\mathrm{OH}$ stretching band broaden $3000 \mathrm{~cm}^{-1}$ to $3600 \mathrm{~cm}^{-1}$ in the IR region. In this region, the peak at $\sim 3450$ $\mathrm{cm}^{-1}$ is assigned to $\mathrm{OH}$ groups hydrogen-bonded to neighbors in a relatively disordered structure. The peak at $\sim 3200 \mathrm{~cm}^{-1}$ is assigned to $\mathrm{OH}$ groups hydrogen-bonded in a well ordered "ice-like" structure [2].

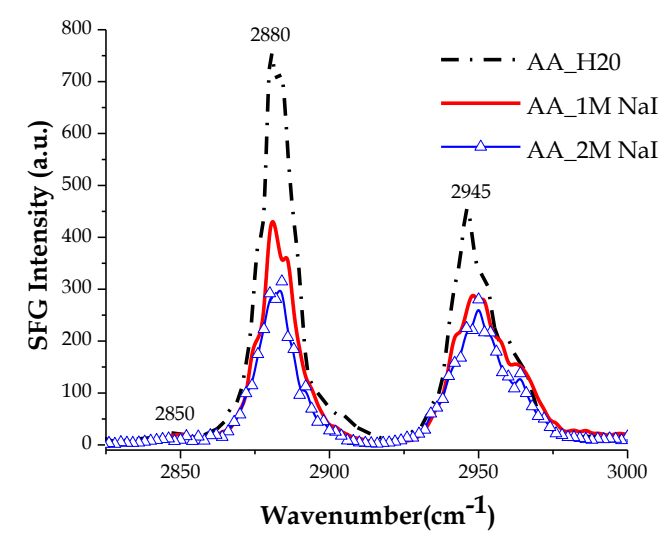

a)

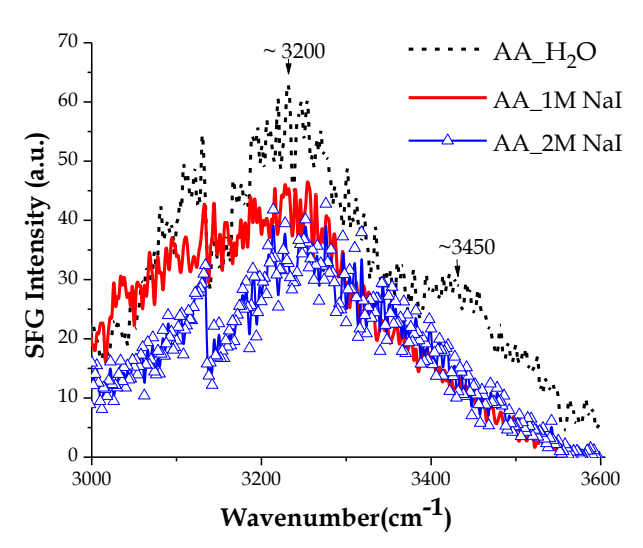

b)

Figure 2. SFG spectra of AA monolayer/ purewater surfaces. 


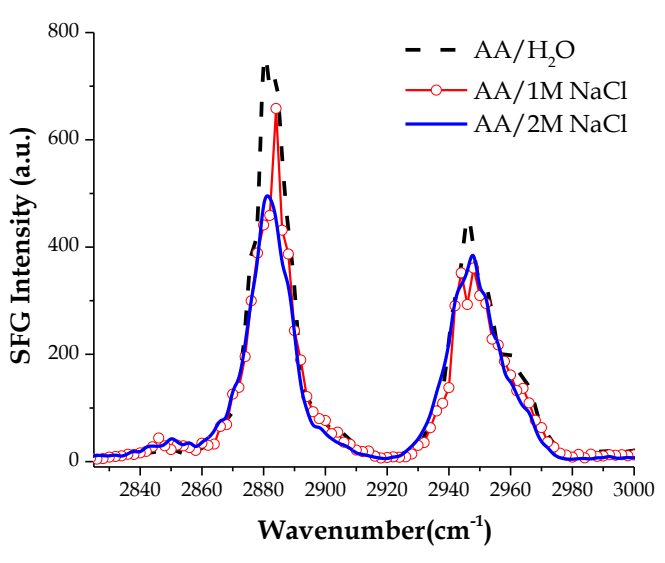

a)

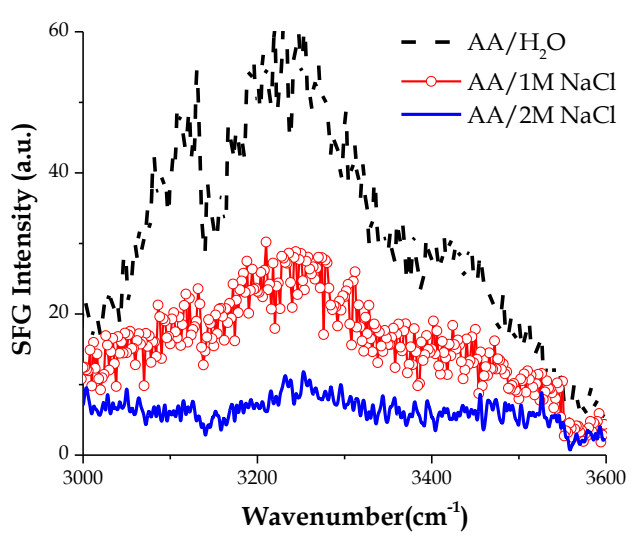

b)

Figure 3. SFG spectra of monolayer AA/ salt solutions $\mathrm{NaCl}$.

We observe a general trend of decreasing SF intensities in presence of salts in the beneath solutions, except for $\mathrm{NaF}$ which will be discussed later. This observation agrees with the previous studies about surface propensities of those relevant ions [4, 5]. In the left panels of Fig. 2 and Fig. 3, we can observe a decreasing of SFG signals in the $\mathrm{CH}$ band in presence of $\mathrm{NaI}$ and $\mathrm{NaCl}$ in the solutions in compare to those from the AA over pure water. Y. R. Shen et. al. [8] have used phasesensitive SFVS to investigate surface propensities of various ions in salt solutions and found a decreasing trend in surface propensities of the ions in following order: $\mathrm{I}^{-}, \mathrm{Cl}^{-}$, and $\mathrm{Na}^{+}$[4]. Molecular dynamic (MD) simulations have also predicted similar results [5].

In our experiment, the decreasing of SF signals in the $\mathrm{CH}$ band indicates that the presence of $\mathrm{I}^{-}$, $\mathrm{Cl}^{-}$at the interfaces disturbs the well-order network of AA molecules of the monolayer on the water. In the meantime, anions $\mathrm{I}^{-}, \mathrm{Cl}^{-}$also disturb the hydro-bonding network of the AA's head-groups with the water molecules at the interfaces, leading to the decreasing of SF intensities in the ice-like band ( $3200 \mathrm{~cm}^{-1}$ ) as seen in Fig. 2b, and Fig. 3 b.

For more detail, we compare effect of $\mathrm{I}^{-}, \mathrm{Cl}^{-}$ions on the interfacial structures of the AA monolayer on solutions of $\mathrm{NaI}$ and $\mathrm{NaCl}$ with the same salt concentration of $1 \mathrm{M}$, as indicated in Fig. 4. As seen in Fig. 4a, the peak intensities of $\mathrm{CH}_{3 \mathrm{ss}}$ and $\mathrm{CH}_{3 \mathrm{Fr}}$ from the $\mathrm{AA}$ monolayer on the $\mathrm{NaI}(1 \mathrm{M})$ solution are lower than that from the $\mathrm{AA}$ on the $\mathrm{NaCl}(1 \mathrm{M})$ solution. This difference can be explained by the larger size and more polarizability of $\mathrm{I}^{-}$anions in compare to those of the $\mathrm{Cl}^{-}$, or the $\mathrm{I}^{-}$ions have a larger surface propensity in relative to the $\mathrm{Cl}^{-}$.

In Fig. 4b, we observed a reversed trend of effects of $\mathrm{I}^{-}$and $\mathrm{Cl}^{-}$on the $\mathrm{OH}$ vibrational band in compare to those on the $\mathrm{CH}$ band. The SF intensity of $\mathrm{OH}$ "ice-like" vibrations $\left(\sim 3200 \mathrm{~cm}^{-1}\right)$ in the interface of $\mathrm{NaI}$ solution is larger than that from the interface of $\mathrm{NaCl}$ solution. For this observation, we propose an explanation as following: due to their higher surface propensity, $\mathrm{I}^{-}$ions "emerge" from the top-most interfacial layers with a larger number in compare to $\mathrm{Cl}^{-}$ions, this point turn out to be agreed with a recent MD simulation result [5]. Thus, these $\mathrm{I}^{-}$ions will have a larger effect on the AA molecular network, and the $\mathrm{Cl}^{-}$ions, on the other hand, disturb the ice-like structure of the water interfacial layers below leading to a decreasing of the SF signal from this structure. 


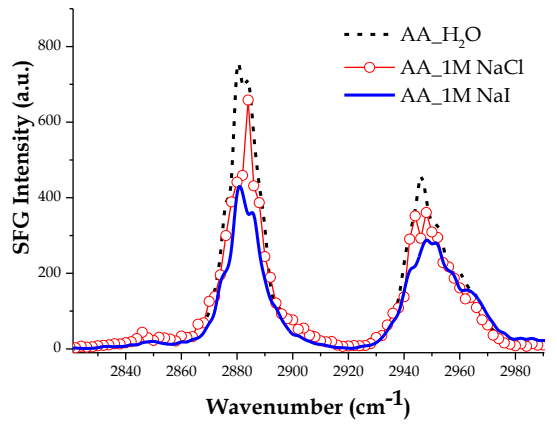

a)

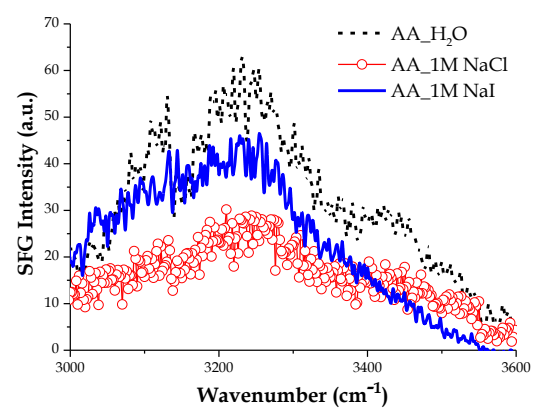

b)

Figure 4. SFG spectra of monolayer $\mathrm{AA} /$ salt solutions $\mathrm{NaI}, \mathrm{NaCl}$ at the same concentration.

We observe a contrary effect of $\mathrm{F}^{-}$on SF spectra from AA monolayer on NaF solutions. In Fig. 5a, the peak intensities of $\mathrm{CH}_{3 \mathrm{ss}}$ and $\mathrm{CH}_{3 \mathrm{Fr}}$ are almost unchanged in presence of $\mathrm{NaF}$ in the solutions with concentration ranging from 0 to $0.8 \mathrm{M}$ (notice that the saturation concentration of $\mathrm{NaF}$ is $\sim 1 \mathrm{M}$ ). However, the SF intensities from $\mathrm{OH}$ "ice-like" bands are significantly increased in the presence of $\mathrm{NaF}$ in solutions, as seen in Fig. 4b. Results from molecular dynamic studies of Pavel Jungwirth et. al. [5] predicted that $\mathrm{F}^{-}$ions in the solution submerge in the top-most water interfacial layer. These ions interact with water molecules at the interfacial layers enhancing the hydrogen bonding network of water at the interface. This result is also in agreement with those from Richmond et. al. [6], which showed that $\mathrm{F}^{-}$anions do not emerge from the topmost layer of an air/water interface and have a "structure-making" characteristic that enhances the water network at the interface.

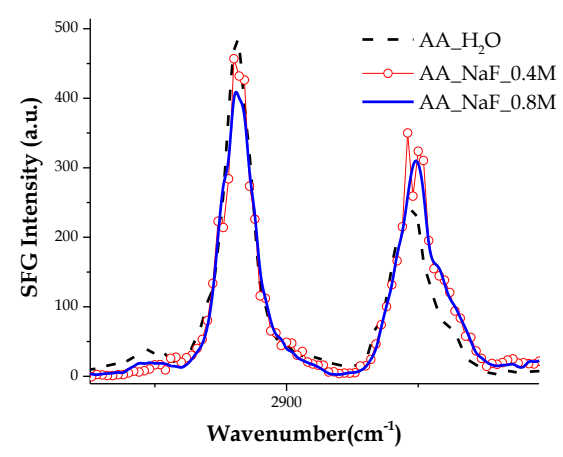

a)

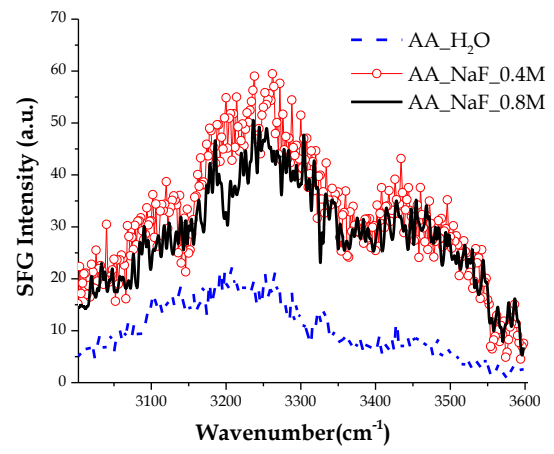

b)

Figure 5. SFG spectra of monolayer AA/ salt solutions NaF.

\section{Conclusions}

In this paper, we use sum-frequency generation vibrational spectroscopy to study effects of various halide anions in the AA Langmuir monolayer on water interface. The results show that those anions 
affect the interface in different ways. Both $\mathrm{I}^{-}$and $\mathrm{Cl}^{-}$ions have high surface propensities and disturb structures at the interface, but due to its higher surface propensity, the $\mathrm{I}^{-}$shows more effect on the monolayer structure, whereas the $\mathrm{Cl}^{-}$shows more effect on the "ice-like" structure of the beneath water. The presence of fluoride $\left(\mathrm{F}^{-}\right)$, on the other hand, shows an enhancement of the hydrogen bonding of water at the interface. These results reinforce similar arguments of some recent studies obtained by molecular dynamic simulation as well as by phase-sensitive SFG-VS.

\section{References}

[1] D. Myers, Surfaces, Interfaces, and Colloids: Principles and Applications, Wiley-VCH Publishers, New York, 1999.

[2] Du, Q.; Superfine, R.; Freysz, E.; Shen, Y. R., Vibrational Spectroscopy of Water at the Vapor/Water Interface, Physical Review Letters 70 (1993) 2313.

[3] P.Guyot - Sionnest, J.H.Hunt, and Y.R.Shen, Sum - Frequency Vibrational Spectroscopy of a Langmuir Film: Study of Molecular Orientation of a Two Dimensional System, Physical Review Letters 59 (1987) 1597.

[4] Chuanshan Tian, Steven J. Byrnes,Hui-Ling Han, and Y. Ron Shen, Surface Propensities of Atmospherically Relevant Ions in SaltSolutions Revealed by Phase-Sensitive Sum Frequency VibrationalSpectroscopy, Journal Physical Chemistry Letters 2 (2011) 1946.

[5] Pavel Jungwirth, and Douglas J. Tobias, Ions at the Air/Water Interface, Journal Physical Chemistry B 106 (2002) 6361.

[6] Elizabeth A. Raymond and Geraldine L. Richmond, Probing the Molecular Structure and Bonding of the Surface of Aqueous Salt Solutions, Journal Physical Chemistry B 108 (2004) 5051. 